\title{
Assessment and Comparison of the Head Posture and Craniofacial Growth in Vertical Dimension- A Cephalometric Study
}

\author{
Anshuka ${ }^{1}$, Usha Shenoy ${ }^{2}$, Sujoy Banerjee ${ }^{3}$, Pooja Wajekar ${ }^{4}$, Varsha Vasvani ${ }^{5}$ \\ ${ }^{1}$ Department of Orthodontics, VSPM Dental College and Research Centre, Nagpur, Maharashtra, India. \\ ${ }^{2}$ Department of Orthodontics, VSPM Dental College and Research Centre, Nagpur, Maharashtra, India. \\ ${ }^{3}$ Department of Orthodontics, VSPM Dental College and Research Centre, Nagpur, Maharashtra, India. \\ ${ }^{4}$ Department of Orthodontics, VSPM Dental College and Research Centre, Nagpur, Maharashtra, India. \\ ${ }^{5}$ Department of Orthodontics, VSPM Dental College and Research Centre, Nagpur, Maharashtra, India.
}

\section{ABSTRACT}

\section{BACKGROUND}

The aim of the present study was to examine as to whether any association can be found between the head posture and the craniofacial growth in the vertical direction.

\section{METHODS}

The sample comprised of 150 subjects in the age group of 18 - 25 years and were further divided into three groups. These groups were classified into hypodivergent, normodivergent, and hyperdivergent according to the sella-nasion (SN) mandibular plane angle. The head posture was measured by calculating craniocervical and cervicohorizontal postural variables, recorded from the lateral cephalograms taken with the subjects standing with the head in the natural head position.

\section{RESULTS}

A clear pattern of association was found between the head posture and the vertical growth pattern. An extended head posture was seen in hyperdivergent group and flexion of the head was seen in hypodivergent group when compared to normodivergent cases $(\mathrm{P}<0.05, \mathrm{P}<0.01)$. The findings were in agreement with the soft tissue stretching hypothesis according to which stronger forces are exerted on the facial skeleton whenever there is an increase in the tension in the soft tissue layer. When these forces are active for a long time during growth, they might restrict the growth of the maxilla and the mandible in forward direction and redirect it in a more caudal direction. Such a mechanism could explain the association between the extension of the head and the development of facial skeleton and in particular, the mandible.

\section{CONCLUSIONS}

The cervical vertebral column area should be evaluated in routine cephalometric analysis and any deviation in the cervical column morphology and head posture should be registered. These registrations may prove useful when considering the diagnosis and evaluating the etiology, especially in patients with severe skeletal malocclusion and obstructive sleep apnea.

\section{KEY WORDS}

Head Posture, Craniofacial Growth, Soft Tissue Stretching

\author{
Corresponding Author: \\ Dr. Anshuka, \\ QTR E9, Medical Enclave, \\ Opposite KC Theatres, \\ Bakshi Nagar, Jammu-180001, \\ $J \& K$, India. \\ E-mail: anshukajasrotia@gmail.com
}

DOI: $10.14260 /$ jemds/2020/277

Financial or Other Competing Interests: None.

How to Cite This Article:

Anshuka, Shenoy $U$, Banerjee $S$, et al. Assessment and comparison of the head posture and craniofacial growth in vertical dimension- a cephalometric study. J. Evolution Med. Dent. Sci. 2020;9(15): 1276-1280, DOI:
Submission 13-02-2020,

Peer Review 25-03-2020,

Acceptance 01-04-2020,

Published 13-04-2020. 


\section{BACKGROUND}

The interdependence between the structural condition and the action of movement is well known. In other words, the muscle action, on which the performance of several functions of the organism depends, is determined by the muscleskeletal balance relationship of the body segments observed in the posture called ideal. ${ }^{1}$ The evolution of an upright posture and bipedal walking has been associated with notable changes that characterize many human bones and muscles which include spinal column, skull, pelvis, legs and all the related joints, ligaments and muscles. Accordingly the spine developed secondary curves in the lumbar and cervical vertebrae, the size of the vertebrae increased from top down, the rib cage flattened and the change in the relative size of the cranium and the jaw allowed the balance of the head to shift backward requiring less powerful muscles on the back of the neck.

The most noteworthy changes that accompanied the development of bipedal stance were that in the cranial base. The cranial base plays a key role in the craniofacial growth, helping to integrate, spatially and functionally, different patterns of growth in various adjoining regions of the skull such as the components of brain, the nasal cavity, the oral cavity and the pharynx. In addition the cranial base connects the skull with the vertebral column and with the mandible, and in this role it is able to influence ontogenetic and interspecific patterns of variation in craniofacial morphology. ${ }^{2}$ An association of the horizontal and vertical dimensions of the atlas vertebra (C1), with head posture, cranial base angulation, and mandibular shape and growth have been shown in various cephalometric studies. ${ }^{3}$ Also, the dimensions of the first cervical vertebra (C1), atlas, as well as the posture of the head and neck are associated with factors such as craniofacial morphology, including the cranial base. ${ }^{3}$ Malformation of the upper cervical vertebrae morphology have been found in patients with condylar hypoplasia, ${ }^{4}$ adult orthodontic surgical patients with skeletal deep bite, ${ }^{5}$ skeletal mandibular overjet, ${ }^{6}$ skeletal horizontal overjet, and skeletal open bite The relationship between head posture and craniofacial morphology was earlier investigated by Schwarz ${ }^{7}$ who contended that extension of the head led to the development of Class II malocclusion. An improved understanding of the co-ordinating mechanisms that contribute to normal craniofacial development is of importance for diagnosis and treatment of morphological and functional disturbances in the masticatory system and adjoining structures. A factor that seems to be of relevance in this connection is the relationship between craniofacial skeletal development and the posture of head and neck. ${ }^{8}$ Many practitioners have recognised that subjects with a large mandibular plane inclination and long face morphology are characterised by an extended head posture and a forward inclined cervical column. It is also typical that the subjects with a short face morphology often carry their heads somewhat lowered and have a markedly backward-curved upper cervical spine. ${ }^{8}$

Despite these observations, little is known about the relationship between the head and neck posture and craniofacial development related to different growth patterns. The aim of the present study is to find any association of head posture with craniofacial growth in vertical direction.

\section{METHODS}

The lateral cephalograms of 150 subjects (females and males, ranging in age from (18-25 years) were divided into three groups according to the Steiner's mandibular plane angle i.e. Hyperdivergent group (SN-MP angle $>36$ degrees) Normodivergent group (SN-MP angle 26-36 degrees) Hypodivergent group (SN-MP angle $<26$ degrees) ${ }^{9}$ The subjects with no prior orthodontic treatment, no craniofacial anomalies or systemic muscle or joint disorders; all permanent teeth till second molars erupted and accessibility of a profile radiograph before orthodontic treatment with the first five cervical vertebral units visible were included in the study. The subjects with history of trauma to the cervical vertebrae or history of any previous surgery were excluded from the study. The lateral cephalograms were traced and analyzed to compare the variables by statistical analysis.

For the craniofacial dimensions, the profile radiographs were taken with the teeth in occlusion and in the standardized natural head posture, the mirror position, as described by Siersbaek-Nielsen and Solow ${ }^{10}$. A long mirror was used to accommodate the different inclinations of the head in individuals while taking the radiographs. The radiographs were taken in a cephalostat with a film-to-focus distance of $180 \mathrm{~cm}$ and a film to- median plane distance of $10 \mathrm{~cm}$. No correction was made for the constant linear enlargement of $5.6 \%$. All the cephalometric reference points and measurements were done manually and double checked by another observer. Fifteen variables representing the head posture, cranial base angle and, the vertical and the sagittal craniofacial dimensions were calculated. (fig. 1). The postural angles that were measured in the present study were the craniocervical (NSL/OPT and NSL/CVT), craniovertical (NSL/VER, NL/VER), cervicohorizontal (OPT/HOR, CVT/HOR) and cervical curvature (OPT/CVT) to determine the head posture.

\section{Reference Points or Lines Used in the Cephalometric Analysis}

- NSL - Sella nasion line NL - palatal plane

- Md - mandibular line

- $\quad$ OPT- odontoid process tangent to cv2ap and cv2ip CVT- cervical vertebrae tangent to cv2ap and cv4ip HOR- True horizontal

- VER- true vertical

- Pog - pogonion, the most anterior point on the mandibular symphysis

- $\mathrm{Ba}$ - basion, the most posterior inferior point on the sagittal plane on the anterior border of the foramen magnum.

\section{Reliability}

30 lateral radiographs were selected randomly from the previously evaluated radiographs and were remeasured to 
check the reliability of the variables describing the head posture. The radiographs were again traced manually and the measurements were cross checked. No significant differences were found between the 2 sets of recordings by using paired $t$ tests.

\section{Statistical Analysis}

The data on angular measurements of cranial base and head postures were obtained for subjects in three study groups (table I). The descriptive statistics like mean, standard deviation, median and range were obtained for each of the measurable parameter. The difference in the means of each parameter across three groups was evaluated for statistical significance using one-way analysis of variance (ANOVA). The pair wise analysis was performed using Tukey's posthoc test. The analyses were performed in SPSS ver 20.0 (IBM Inc.) and the significance was tested at 5\% level.

\section{RESULTS}

Table I provides the descriptive statistics for various craniocervical parameters (NSL-OPT and NSL- CVT; NL-OPT and NL-CVT) in three study groups. The difference in the means across groups was significant for the craniocervical parameters NSL-CVT and NL-CVT as indicated by P-value of $<0.05$. For NSL-CVT, the mean in hyperdivergent group was 105.16 (7.74), in normodivergent group was 98.06 (5.11) and in hypodivergent group was 97.83 (6.83). The difference in the means was statistically highly significant with P-value $<0.0001$. The mean for hyperdivergent group was significantly higher than that of normodivergent and hypodivergent means, as observed through pair wise analysis using Tukey's test. For NL-CVT, the mean in hyperdivergent group was 97.46 (9.61), in normodivergent group was 92.63 (4.91) and in hypodivergent group was 92.71 (7.28). The difference in the means was statistically significant with P-value of $0.020(\mathrm{p}<0.05)$. The mean for hyperdivergent group was significantly higher than that of other two groups as observed through pair wise analysis using Tukey's test.

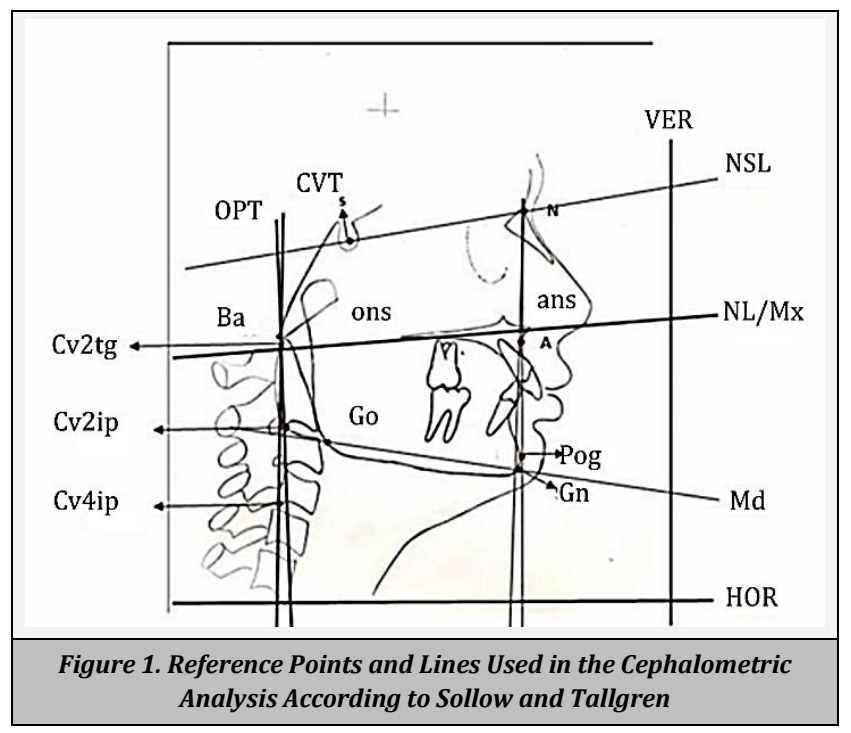

Table II provides the descriptive statistics for craniovertical parameters (NSL-VER and NL-VER) and cervicohorizontal parameters (OPT-HOR and CVT-HOR) for three groups. The difference in the means was statistically significant with P-value of 0.004 using one-way ANOVA for both the craniovertical parameters. The post-hoc analysis using Tukey's test revealed that the mean of craniovertical angles for hyperdivergent group was significantly higher than the other two groups $(\mathrm{P}<0.05)$. For cervicohorizontal parameters, the difference in the means was statistically significant with P-value of

$0.001(\mathrm{p}<0.05)$ using one-way ANOVA. The paired analysis using Tukey's test revealed that the mean for hyperdivergent group was significantly lower than that of normodivergent and hypodivergent groups.

\begin{tabular}{|ccccc|}
\hline Parameters & Groups & Mean & SD & P-Value* \\
& Hyperdivergent & 102.50 & 8.14 & \\
NSL-OPT & Normodivergent & 95.30 & 6.39 & 0.097 \\
& Hypodivergent & 94.87 & 6.85 & \\
& Hyperdivergent & 105.16 & 7.74 & \\
NSL-CVT & Normodivergent & 98.06 & 5.11 & $<0.0001$ \\
& Hypodivergent & 97.83 & 6.83 & \\
& Hyperdivergent & 94.83 & 9.61 & \multirow{2}{*}{ NL-OPT } \\
& Normodivergent & 91.90 & 5.05 & \\
& Hypodivergent & 91.90 & 5.91 & \\
& Hyperdivergent & 97.46 & 9.61 & \multirow{2}{*}{ NL-CVT } \\
& Normodivergent & 92.63 & 4.91 & \\
& Hypodivergent & 92.71 & 7.28 & \\
\hline \multicolumn{5}{c}{ Table 2 } \\
\end{tabular}

\begin{tabular}{|c|c|c|c|c|}
\hline Parameters & Groups & Mean & SD & P-Value* \\
\hline \multirow{3}{*}{ NSL-VER } & Hyperdivergent & 97.03 & 3.42 & \multirow{3}{*}{0.004} \\
\hline & Normodivergent & 94.30 & 4.00 & \\
\hline & Hypodivergent & 94.27 & 3.31 & \\
\hline \multirow{3}{*}{ NL-VER } & Hyperdivergent & 90.40 & 3.31 & \multirow{3}{*}{0.001} \\
\hline & Normodivergent & 88.36 & 3.19 & \\
\hline & Hypodivergent & 91.43 & 2.87 & \\
\hline \multirow{3}{*}{ OPT-HOR } & Hyperdivergent & 84.73 & 6.42 & \multirow{3}{*}{0.001} \\
\hline & Normodivergent & 89.00 & 4.76 & \\
\hline & Hypodivergent & 90.33 & 4.90 & \\
\hline \multirow{3}{*}{ CVT-HOR } & Hyperdivergent & 82.00 & 6.03 & \multirow{3}{*}{0.001} \\
\hline & Normodivergent & 85.56 & 4.07 & \\
\hline & Hypodivergent & 87.56 & 4.98 & \\
\hline \multicolumn{5}{|c|}{$\begin{array}{c}\text { Table 3. Statistics for Craniovertical and Cervicohorizontal } \\
\text { Parameters in the Three Groups }\end{array}$} \\
\hline
\end{tabular}

\section{DISCUSSION}

In this study an attempt is made to find the relationship of head posture and craniofacial growth in vertical dimension. The craniocervical angles (NSL/CVT and NL/CVT) and the craniovertical angles (NSL/VER and NL/VER) were statistically highly significant with P-value $<0.0001$ with an increased value in hyperdivergent group and a decreased value in hypodivergent group. The means of the cervicohorizontal angles (OPT/HOR and CVT/HOR) in the present study were statistically significant with P-value of $0.001(p<0.05)$ using one-way ANOVA. The mean for hyperdivergent group was significantly lower than that of normo divergent and hypodivergent groups. The findings of the present study were in association with the studies done by Solow and Tallgren ${ }^{11}$ who showed that greater cervicohorizontal and small craniocervical angles are associated with a horizontal facial growth pattern characterized by reduced backward displacement of the temporomandibular joint (TMJ), increased growth in length 
of the maxilla, increase in maxillary and mandibular prognathism and larger than average forward true rotation of the mandible. Subjects with lower cervicohorizontal and large craniocervical angles were associated with a vertical facial development and are likely to exhibit large backward displacement of the TMJ, reduced growth in length of the maxilla, reduction of maxillary and mandibular prognathism and less than average forward true rotation of the mandible. The larger the angulation, the more vertical the facial growth pattern in boys (Huggare and Cooke,12 1994) Solow and Tallgren, ${ }^{11}$ 1976; Hellsing et al, ${ }^{13} 1987$; Showfety et al,141987; Leitao and Nanda,15 2000; Solow and Sandham 8,2002 in their studies reported the association of extended head position with increased craniocervical angles, craniovertical angles and decreased cervicohorizontal angles.

Also, the term flexion of the head denotes a forward bent position of the head and was generally associated with backward slope of the cervical column (Solow and Tallgren,11 1976; Solow and Sandham, ${ }^{8}$ 2002), i.e. a vertical cervical posture (Ozbek and Koklu,16 1993) and decreased craniocervical, craniovertical angles and increased cervicohorizontal angles.

It can therefore be concluded that in the present study an extended head posture was seen in hyperdivergent patients and hypodivergent patients had a flexed head posture. An explanation for these associations has been described in the literature as the -soft tissue stretching hypothesis (Solow and Kreiborg, ${ }^{17}$ 1977) as mentioned above. According to the soft tissue stretching hypothesis an extended head posture increases the distance between the mandible and the sternum thereby stretching the soft tissues covering the face and the neck. This increase in the tension of the soft - tissue layer exerts slightly stronger forces on the facial skeleton. When these forces are active for a long time during growth, they might restricts the growth of the maxilla and the mandible in forward direction and redirect it in a more caudal direction. Such a mechanism could explain the association between the extension of the head and the development of facial skeleton and in, particular, the mandible.

So to conclude within limitations, extended head posture was seen in hyperdivergent patients and flexion of the head was seen in hypodivergent patients when compared with the normodivergent group. The hypothetical explanations which are given in the literature with respect to the change in head posture, mandibular growth, maxillary growth as well as change in the cervical column morphology need to be substantiated with further research and if they can be confirmed, it would be beneficial to establish borderline conditions for clinical decisions and to find out the possible etiological factors.

\section{CONCLUSIONS}

An extended head posture is seen in hyperdivergent patients. A flexed head posture is seen in individuals with hypodivergent growth pattern. The cervical vertebral column area should be evaluated in routine cephalometric analysis and any deviation in the cervical column morphology and head posture should be registered. These registrations may prove useful when considering the diagnosis and evaluating the etiology, especially in patients with severe skeletal malocclusion and obstructive sleep apnea.

\section{REFERENCES}

[1] Webwer P, Correa E, Bolzana G, et al. Relationship between craniocervical posture, mandible and hyoid bone and influence on alimentary functions. Braz J Oral Sci 2012:11 (2):141-7.

[2] De Vincenzo JP. Treatment options for sagittal corrections in noncompliant patients. In: Graber TM, Vanarsdall RL, Vig KWL, eds. Orthodontics: Current principles and techniques. $4^{\text {th }}$ edn. St. Louis, MO: Elsevier-Mosby 2005: p. 117.

[3] Sandikcioglu M, Skov S, Solow B. Atlas morphology in relation to craniofacial morphology and head posture. European Journal of Orthodontics 1994;16 (2):96-103.

[4] Sonnesen L, Pedersen CE, Kjær I. Cervical column morphology related to head posture, cranial base angle and condylar malformation. Eur J Orthod 2007;29 (4):398-403.

[5] Sonnesen L, Kjaer I. Cervical vertebral body fusions in patients with skeletal deep bite. Eur J Orthod 2007;29 (5):464-70.

[6] Sonnesen L, Kjaer I. Cervical column morphology in patients with skeletal Class III malocclusion and mandibular overjet. Am J Orthod Dentofacial Orthop $2007 ; 132$

[7] (4):427.e7-e12.

[8] Schwartz AM. Positions of the head and malrelations of the jaws. Int J Orthod 1928;14 (1):56-8.

[9] Solow B, Sandham A. Cranio-cervical posture: a factor in the development and function of the dentofacial structures. Europen Journal of Orthodontics 2002;24 (5):447-56.

[10] Steiner CC. The use of cephalometrics as an aid to planning and assessing orthodontic treatment. American Journal of Orthodontics 1960;46 (10):72135.

[11] Solow B, Siersbaek-Nielsen S. Growth changes in head posture related to craniofacial development. Am J Orthod 1986;89 (2):132-40.

[12] Solow B, Tallgren A. Head posture and craniofacial morphology. Am J Phys Anthropol 1976;44 (3):417-35.

[13] Huggare JA, Cooke MS. Head posture and cervicovertebral anatomy as mandibular growth predictors. European Journal of Orthodontics 1994;16 (3):175-80.

[14] Hellsing E, L'Estrange P. Changes in lip pressure following extension and flexion of the head and at changed mode of breathing. Am J Orthod Dentofacial Orthop 1987;91 (4):286-94.

[15] Showfety KJ, Vig PS, Matteson S, et al. Associations between the postural orientation of sellanasion and skeletodental morphology. Angle Orthodontist 1987;57:99-112. 
[16] Leitao P, Nanda RS. Relationship of natural head position to craniofacial morphology. American Journal of Orthodontics and Dentofacial Orthopedics 2000;117 (4):406-17.

[17] Ozbek MM, Koklu A. Natural cervical inclination and craniofacial structure. Am J Orthod Dentofacial Orthop 1993;104 (6):584-91.
[18] Solow B, Kreiborg S. Soft-tissue stretching: a possible control factor in craniofacial morphogenesis. Scandinavian Journal of Dental Research 1977;85 (6):505-7. 